International Journal of Cancer Studies \& Research (IJCR) $1 S S N: 2167-9118$

\title{
Lung Fibrosis after Chemotherapy Containing Docetaxel in a Patient Treated for Breast Cancer
}

\author{
Case Report
}

A. Hassouni ${ }^{1 *}$, Z. Youbi ${ }^{1}$, N. Tawfiq ${ }^{1}$, Z. Boughaleb ${ }^{1}$, N. Naqos ${ }^{2}$, A. Rachdi ${ }^{2}$, A. Taleb $^{2}$, Z. Bouchbika $^{1}$, N. Benchakroun ${ }^{1}$, H. Jouhadi ${ }^{1}$, S. Sahraoui $^{1}$, A. Benider ${ }^{1}$

${ }^{1}$ MOHAMMED VI Cancer treatment Center, Radiation Oncology Department, Ibn Rochd University Hospital, Morocco.

${ }^{2}$ MOHAMMED VI Cancer treatment Center, Medical Oncology Department, Ibn Rochd University Hospital, Morocco.

\begin{abstract}
Docetaxel has proven effective in many solid tumors, including breast cancer. Hypersensitivity reactions are the most common toxicity associated with docetaxel. However, acute interstitial pneumonia is rarely reported in the literature. We report the case of a woman born in 1969, treated for invasive ductal carcinoma of the left breast and who presented 6 years later with an invasive ductal carcinoma locally advanced in the right breast. Neo-adjuvant chemotherapy based on docetaxel was indicated. Fifteen days after the third course, she had a stage IV dyspnea with a dry cough. Chest radiography showed a bilateral diffuse interstitial infiltrate appearance labeled as an acute interstitial lung disease secondary to docetaxel. The outcome was good after a high dose of corticosteroids. Clinicians should be aware of the risk of interstitial lung disease with docetaxel and introduce a special monitoring of lung function.
\end{abstract}

Keywords: Lung; Fibrosis; Docetaxel; Breast.

\section{*Corresponding Author:}

Abdeslam HASSOUNI,

MOHAMMED VI Cancer treatment Center, Radiation Oncology Department, Ibn Rochd University Hospital, Morocco.

Tel: +212670982624

E-mail: abdes.hassouni@gmail.com

Received: July 08, 2015

Accepted: August 15, 2015

Published: August 18, 2015

Citation: A. Hassouni et al., (2015) Lung Fibrosis after Chemotherapy Containing Docetaxel in a Patient Treated For Breast Cancer. Int J Cancer Stud Res. 4(4), 82-85. doi: http://dx.doi.org/10.19070/2167-91181500012

Copyright: A. Hassouni ${ }^{\odot}$ 2015. This is an open-access article distributed under the terms of the Creative Commons Attribution License, which permits unrestricted use, distribution and reproduction in any medium, provided the original author and source are credited.

\section{Introduction}

Docetaxel is a chemotherapeutic agent which is increasingly used in the treatment of several types of cancer including breast cancer $[1]$.

Its main side effects are neutropenia, mucocutaneous hypersensitivity reactions and fluid retention. Interstitial pneumotistis is rarely reported as a side effect in the literature.

We report the case of a patient with bilateral breast invasive ductal carcinoma who developed acute diffuse interstitial pneumotistis following chemotherapy containing docetaxel.

\section{Case Report}

Ms. AK is a 46 year old female with no signficant past medical history that we were consulted to evaluate for two years of increasing left breast size and progressively worsening mastodynia. Clinical examination noted increased breast volume with a retracted nipple, skin thickening and a nodule of $7 \mathrm{~cm}$ in diameter in the lower inner quadrant associated with inflammation of the skin. The right breast was normal and no axillary or supraclavicular adenopathy were found. The biopsy of the nodule has objectified infiltrating ductal carcinoma grade II SBR. The chest x-ray (Figure 1) and abdominal ultrasound were normal. It was classified T4bNoMo. She received 4 cycles of neoadjuvant chemotherapy according to the AC60 protocol. She underwent a modified radical mastectomy and there was a residual tumor measuring $40 \mathrm{~mm}$ in diameter and a single axillary node was involved. She received two courses of adjuvant chemotherapy according to 5FU/Navelbine protocol. Radiotherapy was delivered on the chest wall, axillary, supraclavicular lymph nodes and internal mammary chain at 50 Gy. She finished her treatment in March 2005. Hormone receptors were positive, and she was put on Tamoxifen for 5 years. She has been regularly monitored in the consultation. The chest $\mathrm{x}$-ray done in August 2005 was normal. The chest x-ray done in November 2006 revealed a difference in transparency between the two lung fields, related to mastectomy without parenchymal lesion. The chest x-ray in October 2007 was normal.

The patient was evaluated in March 2008 for bilateral cervicalbrachial neuralgia. Bone scan objectified a hyperfixation in the cervical spine. MRI confirmed metastatic involvement of D1. The chest x-ray was normal (Figure 2). Abdominal ultrasound was normal. CA $15-3$ was $40.2 \mathrm{U} / \mathrm{ml}$. She received decompressive radiotherapy on the cervical spine from C6 to D3, taking into ac- 
count the dose already received on the spinal cord in the anterior axillary lymph node areas, supraclavicular and internal mammary irradiation, and hormone therapy with exemestane was given. CA 15-3 was $36.4 \mathrm{U} / \mathrm{ml}$ (down 11\%) after two months of endocrine therapy. Two months later, the CA $15-3$ was $58.5 \mathrm{U} / \mathrm{ml}$. A bone scan showed regression of uptake on $\mathrm{T} 1$ and no new sites of uptake.

\section{The CA 15-3 has remained at high levels despite normal im- aging}

In March 2010, she had skin thickening with orange peel on the lower quadrant of the right breast associated with homolateral axillary adenopathies. The mammography-sonography noted diffuse thickening of the right breast. A skin biopsy found a moderately differentiated CCI SBR II associated with vascular emboli. The staging was normal including a chest X-ray Normal. Bone scan did not found new sites of uptake. The abdominopelvic and neck ultrasonography were normal. It was classified T4bN2M0. Neoadjuvant chemotherapy with docetaxel at a dose of $75 \mathrm{mg} /$ $\mathrm{m}^{2}$ and capecitabine at a dose of $2000 \mathrm{mg} / \mathrm{m}^{2}$ has been started. An evaluation was planned after 3 cycles. The surgery was scheduled after regression of inflammatory signs on the breast and of the right axillary adenopathies. The first two treatments were well tolerated by the patient.

Fifteen days after the third treatment (21 June 2010), she was evaluated for major dyspnea stage IV associated with a dry cough and asthenia. The Chest x-ray showed bilateral diffuse interstitial infiltrate suggestive of pulmonary metastatic spread. Faced with respiratory failure, no treatment has been decided on the oncological plan. She was put on corticosteroid therapy of $60 \mathrm{mg}$ daily for 6 days. Given the persistence of dyspnea, corticosteroid dose was increased to $120 \mathrm{mg}$ per day orally for a period of one week. The patient was seen a week later. In the absence of respiratory function improvement metastatic lung lesions character were retained and no treatment was decided. Faced with respiratory failure, no treatment has been decided on the oncological plan. She was put on corticosteroid therapy to $60 \mathrm{mg}$ for 6 days. Given the persistence of dyspnea, the corticosteroid dose was increased to 120 mg per day orally for a period of one week. The administration of an oxygen therapy and a high dose pf corticosteroid followed by gradual regression for 2 months, have led to a complete regression of the dyspnea. A radiological control showed the organization of pulmonary involvement in a visible proximal interstitial infiltrate on a chest CT (Figure 3).

The surgery of the right breast was cancelled due to the inflammatory interstitial lung disease. The patient received radiotherapy to the right breast, axillary, and supraclavicular nodes at a dose of 50 Gy from January to March 2011. She had a modified radical mastectomy in April 2011 in front of the improvement of the respiratory function. In the histological study, there was a chronic fibrous reshuffle with less than $5 \%$ of residual tumor post-radiotherapy and a single adenopathy was involved in the axillary dissection from 10 lymph node collected.

Hormone receptors were negative. The HercepTest was positive $3+$. Herceptin was not administered because of the risk of heart failure when she already had a lung injury. In August 2011, the patient presented bilateral cervicobrachial neuralgia with paresis of both upper limbs. MRI of the thoracic spine revealed cord compression at the level of T1 Radiotherapy was already issued on this site at a dose of $45 \mathrm{~Gy}$ in 2008. A decompressive laminectomy sighting was proposed. The lesion was considered a slowly progressive neurological level by neurosurgeons with a risk of addiction to artificial respiratory ventilation after the surgery. Thus, surgery was deferred. An exploration of respiratory function was performed in December 2011 to evaluate the respiratory risk during anesthesia. Bronchoscopy showed a normal aspect of the bronchi. The pulmonary function noted normal flow-volume curve. The diagnosis was that of sequelae after chemotherapy damage. She was put on oral cyclophosphamide for 3 months without response and oral capecitabine for 3 months without response. Faced with the worsening cervicobrachial neuralgia in December 2012, an abdominothoracic and pelvic CT has been requested and showed D1 body compaction fracture with significant lysis of the posterior arch isolated without other associated visceral or bone lesions. In the lung, it revealed diffuse bilateral not septal thickening in both upper lobes and a frosted glass appearance with retraction of both lungs, thickened peri-pulmonary vascular bilateral diffuse evoking a post-treatment pulmonary fibrosis. CA 15-3 has reached 131.6 U/ml. She had the decompressive laminectomy on 2 January 2013 with uneventful postoperative particularly in respiratory function. Histological study of bone and epidural samples showed epidural and bone metastasis of poorly differentiated breast adenocarcinoma. A month later, she developed an inflammatory relapse at the right wall. Echocardiography made as part of the evaluation of the pre-chemo heart function showed an ejection fraction of the left ventricle kept at $70 \%$, a concentric left ventricular hypertrophy and especially the absence of pulmonary hypertension stigma cardiac impact of prior post-chemotherapy lung damage.

\section{Discussion}

Docetaxel is an antineoplastic agent that inhibits the growth of tumors by inducing a stabilization of microtubules and promot-

Figure 1. Chest $\mathrm{x}$-ray of the first staging in 2004 showing normal lungs.

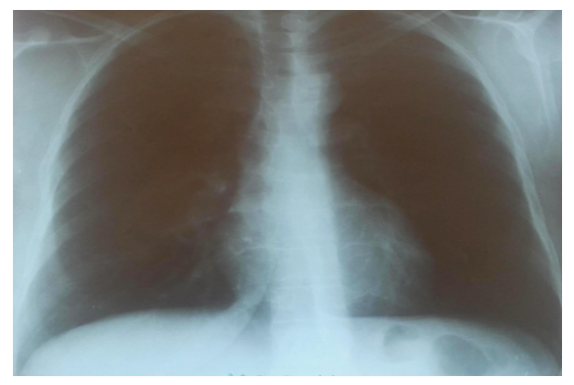


Figure 2. Chest x-ray in 2008 for staging in the bone relapse of breast cancer showing normal lungs.

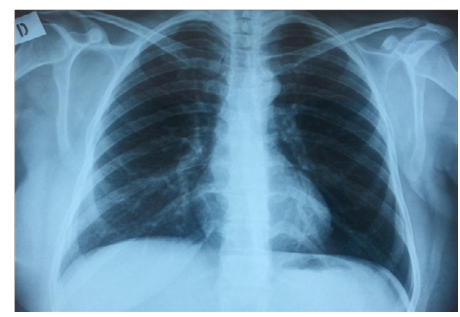

Figure 3. Chest CT after 2 months of corticostoreid showing improvement of the IP.

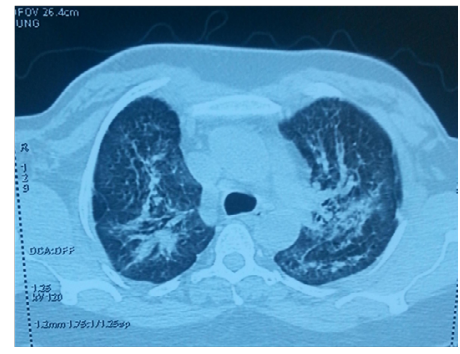

ing the inactivation of bcl-2, thus sensitizing malignant cells to apoptotic stimuli [2]. It has proven its effectiveness in many solid tumors, including breast, stomach, ovaries, and NSCLC [1].

Hypersensitivity reactions are the most common toxicity associated with Docetaxel.

Pulmonary toxicity is more known with Paclitaxel [3, 4]. It usually occurs 48 hours after injection and disappears after a period of 24-96 hours after initiation of corticosteroid therapy.

There are few data in the literature associating interstitial pneumotistis (IP) with Docetaxel. Symptoms usually occur later and last longer. The evolution can be fatal as reported in some clinical cases. Read et al [5] reported 4 cases of IP occurring 1-2 weeks after administration of Docetaxel and have all used mechanical ventilation due to acute respiratory failure.

The symptoms are variable and non-specific. Patients may present with a flu syndrome, a mild fever, dry cough and also dyspnea. Often these symptoms are interpreted as an infection and treated with broad spectrum antibiotics, without clinical improvement [6].

Chest X-ray can show ground-glass opacity. A chest CT scan is much more sensitive and typically shows reticular or nodular or disseminated bilateral reticulo-nodular infiltrates [7]. However, the diagnosis can not be made solely on imaging.

Breast cancer frequently gives pulmonary metastasis. It is therefore necessary to take a biopsy during bronchoscopy with analysis of the aspirated fluid to eliminate a differential diagnosis [8].

IP induced by a drug is marked in the aspirated fluid by lymphocytic alveolitis, an increase in the number of total cells, an increase in the proportion of neutrophils and eosinophils, and a decreased CD4/CD 8 count and in the trans- bronchial biopsy by edema and thickening of the alveolar septa and alveolar and interstitial infiltration of mononuclear cells with an intraluminal organization and aggregation of alveolar macrophages [8]. In our case, the patient had no bronchoscopy but clinical and radiological evolution after the introduction of high-dose corticosteroid therapy was in favor of a toxic origin associated with Docetaxel.

IP usually occurs after the second cycle of Docetaxel and can also occur between 1 and 9 treatment cycles. The mortality of Interstitial fibrosis is estimated at 40\% [5]. In this case, the patient developed IP after the third cycle of Docetaxel.

Pre-existing pulmonary emphysema (p: 0.016) or IP $(\mathrm{P}<0.05)$, is accompanied by an increased risk of developing pulmonary toxicity. The authors concluded not to start Docetaxel if they knew of pre-existing IP [9].

The symptoms rapidly increase for 1 to 2 days and can result in respiratory failure requiring mechanical ventilation [10].

The treatment of choice is the administration of corticosteroids type prednisolone at a dose of 30-60mg daily for 2 to 3 weeks and even $60-240 \mathrm{mg}$ daily for severe cases such as acute respiratory failure [10]. In our case, the patient used a high dose corticosteroid $120 \mathrm{mg}$ per day with gradual regression in two months.

Docetaxel is largely used for the treatment of localized and metastatic breast cancer. However, clinical and radiological monitoring of respiratory function is recommended for the early diagnosis of IP and to prevent progression to an acute respiratory failure that can threaten the vital prognosis of the patient. Particular caution should be taken for patients with pre-existing respiratory disease, in particular interstitial disease. An evaluation of lung function should be recommended before starting treatment with taxanes to prevent aggravation of their respiratory function. The search for IP predisposing factors should help in selecting patients who can not be exposed to taxanes in their oncology treatment plan.

\section{References}

[1]. Haldar S, Basu A, Croce CM (1997) Bcl2 is the guardian of microtubule integrity. Cancer Res 57(2): 229-233.

[2]. Polyzos A, Syrigos K, Stergiou J, Panopoulos C, Potamianou A, et al. (2005) Phase I trial of weekly docetaxel with a 4-weekly cisplatin administration in patients with advanced gastric carcinoma. Cancer Chemother Pharmacol 55(5): 466-470.

[3]. Khan A, McNally D, Tutschka PJ, Bilgrami S (1997) Paclitaxel induced 
acute bilateral pneumonitis. Ann Pharmacother 31(12): 1471-1474.

[4]. Ramanathan RK, Reddy VV, Holbert JM, Belani CP (1996) Pulmonary infiltrates following administration of paclitaxel. Chest 110(1): 289-292.

[5]. Read WL, Mortimer JE, Picus J (2002) Severe interstitial pneumonitis associated with docetaxel administration. Cancer 94(3): 847-853.

[6]. Cordier JF, Loire R, Brune J (1989) Idiopathic bronchiolitis obliterans organizing pneumonia. Definition of characteristic clinical profiles in a series of 16 patients. Chest 96(5): 999-1004.

[7]. Fraser RS, Müller NL, Coleman CD, Paré PD (1999) Fraser and Paré's Diagnosis of Diseases of the Chest. (4th edtn), Saunders, Philadelphia.
[8]. Min BD, Kang HW, Kim WT, Kim YJ, Yun SJ, et al. (2012) DocetaxelInduced Fatal Interstitial Pneumonitis in a Patient with Castration-Resistant Prostate Cancer. Korean J Urol 53(5): 371-374.

[9]. Tamiya A, Naito T, Miura S, Morii S, Tsuya A, et al. (2012) Interstitial lung disease associated with docetaxel in patients with advanced non-small cell lung cancer. Anticancer Res 32(3): 1103-1106.

[10]. Nagata S, Ueda N, Yoshida Y, Matsuda H, Maehara Y (2010) Severe interstitial pneumonitis associated with the administration of taxanes. J Infect Chemother 16(5): 340-344. 